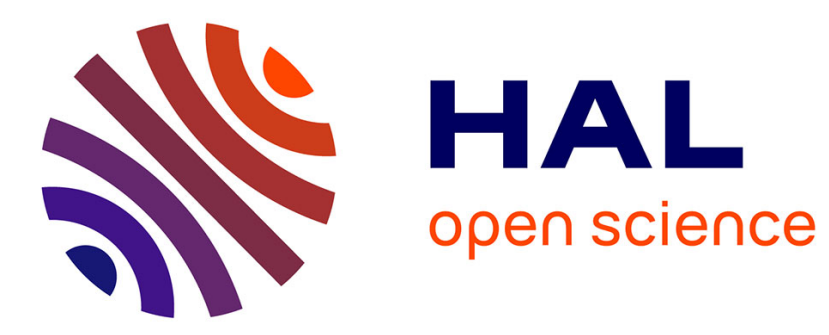

\title{
Principles of Bragg-Fresnel multilayer optics
}

V.V. Aristov, A.I. Erko, V.V. Martynov

\section{To cite this version:}

V.V. Aristov, A.I. Erko, V.V. Martynov. Principles of Bragg-Fresnel multilayer optics. Revue de Physique Appliquée, 1988, 23 (10), pp.1623-1630. 10.1051/rphysap:0198800230100162300 . jpa00245991

\section{HAL Id: jpa-00245991 https://hal.science/jpa-00245991}

Submitted on 1 Jan 1988

HAL is a multi-disciplinary open access archive for the deposit and dissemination of scientific research documents, whether they are published or not. The documents may come from teaching and research institutions in France or abroad, or from public or private research centers.
L'archive ouverte pluridisciplinaire HAL, est destinée au dépôt et à la diffusion de documents scientifiques de niveau recherche, publiés ou non, émanant des établissements d'enseignement et de recherche français ou étrangers, des laboratoires publics ou privés. 
Classification

Physics Abstracts

$42.78 \mathrm{H}-61.10-78.65$

\title{
Principles of Bragg-Fresnel multilayer optics
}

\author{
V. V. Aristov, A. I. Erko and V. V. Martynov \\ Institute of Problems of Microelectronics Technology and Superpure Materials, U.S.S.R. Academy of \\ Sciences, 142432 Chernogolovka, Moscow District, U.S.S.R.
}

(Reçu le 29 septembre 1987, accepté le 8 février 1988)

\begin{abstract}
Résumé. - Cet article décrit les principes et les modèles théoriques de nouveaux éléments optiques utilisant à la fois la diffraction de Bragg et celle de Fresnel. La diffraction de Bragg sur les plans successifs d'une multicouche permet d'obtenir une meilleure résolution spatiale que celle des optiques à miroirs conventionnels plans ou courbes. Les optiques de Bragg-Fresnel combinent les avantages de la bonne résolution spectrale des optiques à réseaux de type Fresnel avec la stabilité des miroirs multicouches.
\end{abstract}

Abstract. - The paper describes the principles and theoretical models of new X-ray optical elements based on the behaviour of Bragg-Fresnel diffraction. The use of volume diffraction permits one to achieve better spatial resolution compared with conventional plane optics and bending mirrors. The construction of Bragg-Fresnel elements combines the advantages of high-resolution Fresnel optics with stability of multilayer mirrors.

\section{Introduction.}

The first successful attempts of X-ray beam focusing were associated with the achievements in producing smooth surfaces of a required shape. This led to the development of grazing incidence X-ray optics [1-3]. However, strong aberrations brought about by grazing incidence confined the resolving power to several microns. The progress of microfabrication technology during the past decade initiated a new stage in the development of X-ray optics, namely, plane optics on the basis of Fresnel zone plates $[4,5,6]$. At present, the minimum dimensions of zones of such plates reach $\sim 50 \mathrm{~nm}$ [7]. This resolution is apparently close to the ultimate one, since in the $\mathrm{X}$-ray wave length range the plane optics approximation is not quite correct, especially for phase elements. In the nanometric wavelength band refractive index differs from unit by $10^{-2}-10^{-3}$. The thickness $t$ of the material required to change the transmitted radiation phase by $\pi$ rad increases up to several microns. As a result, X-ray optical elements become three-dimensional, hence, the plane optics approximation is inapplicable for the nanometric wavelenth band. New possibilities of wave front formation should be considered taking into account the threedimensional diffraction phenomena. Fabrication of multilayer X-ray mirrors was a considerable step forward. The recent progress in their production enabled mirror optics aberrations to be appreciably decreased owing to approximation of incidence angles to a normal one [8].

All the achievements in the production of smooth surfaces, multilayer interference coatings and structures with submicron element dimensions allow for switching over to a new stage of X-ray optics development, namely, fabrication of volume optical elements with three-dimensional Fresnel zone structures. Bragg-Fresnel optics elements permit bringing the practical limit of resolution closer to the diffraction limit, $\sim \lambda$.

Aberrations of optical elements can be almost completely eliminated, in a sufficiently wide aperture, by fabricating a relief with submicron element dimensions in a multilayer interference mirror on the substrate of a prearranged shape. A basic distinction of Bragg-Fresnel element optics is the use of coherent Bragg scattering by separate layers of the material. This makes it possible to increase their diffraction efficiency, reduce chromatic aberrations, accomplish phase and amplitude modulation of radiation and produce switched X-ray optical elements.

Table I presents comparative data on the potentialities of various types of X-ray optical elements.

\section{Principles of Plane Focusing Diffraction Optics.}

Let us describe the principles of focusing diffraction optics beginning with the scheme in figure 1 . It 
Table I.

\begin{tabular}{|c|c|c|c|}
\hline \multicolumn{2}{|c|}{$\begin{array}{c}\text { Grazing incidence } \\
\text { optics } 1952\end{array}$} & \multicolumn{2}{|r|}{ Bragg-Fresnel optics 1986} \\
\hline \multicolumn{2}{|l|}{ Optical scheme $\underbrace{\mathrm{A}_{1}}_{k p}$} & & ${ }^{\mathrm{A}_{1}}$ \\
\hline & $\varphi_{k p}=\sqrt{\Delta n}$ & $f=\frac{r_{1}^{2}}{\lambda} ; r_{N}=N \lambda f+\frac{N^{2} \lambda}{4}$ & $\underline{\lambda} ; N-1<\frac{\left[\mathbf{k}_{1} \cdot \mathbf{r}\right]^{2}}{r_{1}}+\frac{\left[\mathbf{k}_{2} \cdot \mathbf{r}\right]^{2}}{r_{2}} \leqslant N$ \\
\hline Chromatic aberrations & - & $\begin{array}{lll}\lambda & \mathrm{r} \\
\mathrm{r} & \mathrm{r}\end{array}$ & $\begin{array}{l}\text { none, the image is formed in a } \\
\text { reflected wavelength range }\end{array}$ \\
\hline $\begin{array}{l}\text { Limiting resolution } \\
\qquad a_{\max }\end{array}$ & $\begin{array}{l}\text { limiting by strong } \\
\text { aberrations at a } \\
\text { grazing incidence } \\
\sim 1 \mu \mathrm{m}\end{array}$ & $\begin{array}{c}50-100 \mathrm{~nm} \\
a_{\max }=2 \lambda /(1-n)^{1 / 2}\end{array}$ & $\begin{array}{l}\text { determined by technological pos- } \\
\text { sibilities and reaches } 10 \mathrm{~nm} \text {. } \\
\text { when using bending substrates }\end{array}$ \\
\hline $\begin{array}{l}\text { Maximum angular } \\
\text { aperture } \varphi_{\max }\end{array}$ & $\varphi_{\max }<\varphi_{k p}$ & $\varphi_{\max }=\frac{1}{2}(1-n)^{1 / 2}$ & $\begin{array}{l}\text { determined by technological } \\
\text { possibilites }\end{array}$ \\
\hline $\begin{array}{l}\text { Possibility of amp- } \\
\text { litude and phase } \\
\text { modulations }\end{array}$ & none & none & possible \\
\hline $\begin{array}{l}\text { Possibility of hybrid } \\
\text { scheme fabrication }\end{array}$ & none & none & $\begin{array}{l}\text { switching and controlling of an } \\
\mathrm{X} \text {-ray signal by electrical and } \\
\text { sound signals is possible }\end{array}$ \\
\hline $\begin{array}{l}\text { Possibility of fabri- } \\
\text { cation of different } \\
\text { X-ray optical } \\
\text { elements }\end{array}$ & $\begin{array}{l}\text { fabrication of } \\
\text { focusing and } \\
\text { dispersive ele- } \\
\text { ments is possible }\end{array}$ & $\begin{array}{l}\text { fabrication of } \\
\text { lenses and gra- } \\
\text { tings is possible }\end{array}$ & $\begin{array}{l}\text { fabrication of lenses, modula- } \\
\text { tors and mirrors is possible }\end{array}$ \\
\hline
\end{tabular}

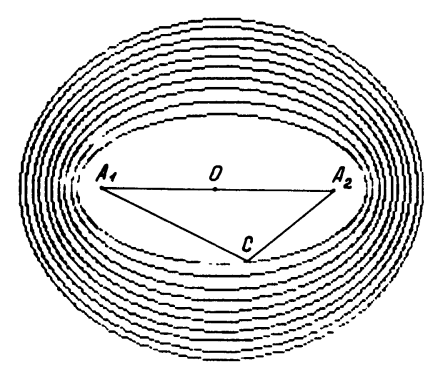

Fig. 1. - Rotational ellipsoids which represent equal phase surfaces relative to the points $A_{1}$ and $A_{2}$.

shows an interference picture of two spherical waves radiated from the $A_{1}$ and $A_{2}$ points. Each line in figure 1 is a plane cross-section of a phase surface represented as rotational ellipsoids with the poles located at the point $A_{1}$ and $A_{2}$. Any scattering point $\mathrm{C}$ belonging to a given surface of an ellipsoid gives the same wave phase under observation at the point $A_{2}$ (if the point $A_{1}$ is a source). To clarify the following description, phase shift between the surfaces of the ellipsoids in figure 1 is chosen to be equal to $\pi$ (path difference is $\lambda / 2$ ). Now let us assume that an opaque plane screen $\mathrm{K}$ is situated between the point $A_{1}$ (a source) and $A_{2}$ (an image) perpendicular to the axis $A_{1} A_{2}$ of the scheme (Fig. 2).

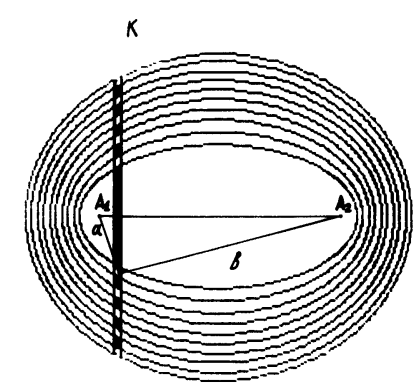

Fig. 2. - Scheme of transparent Fresnel zone plate formation using ellipsoids of an equal phase.

We can obtain a Fresnel zone plate by cutting the opaque screen $\mathrm{K}$ at corresponding places. It will occur if we open only those sections of the screen which make a contribution with one and the same phase value into the image at the point $A_{2}$. Radius of the $n$-th Fresnel zone (Fig. 2) is given by the expression (ignoring a square term by $\sim \lambda^{2}$ ) :

$$
r_{n}=\left(n \frac{a b}{a+b} \lambda\right)^{1 / 2}
$$

where $n$ is the zone number. The total aperture of the zone plate $A=2 r_{N}, a$ and $b$ are corresponding distances to the source and image.

If we use the formula of a thin lens $\frac{1}{a}+\frac{1}{b}=\frac{1}{F}$, 
expression (1) can be written in the form : $r_{n}=(F n \lambda)^{1 / 2}$, where $F$ is a focal distance.

It is useful to show several practical consequences of this formula which are used when calculating parameters of real zone plates. The total aperture of the zone plate is determined by the expression :

$$
A=\frac{F \lambda}{\delta r_{n}} .
$$

Here $\delta r_{n}$ is a minimum width of the of the $n$-th zone, which can be fabricated by the given technique. It should be noted that a spatial resolution of zone optics is $\delta_{1} \sim \delta r_{n}$ and in the range of soft X-ray radiation is limitted by a number of physical and technological causes. Among technological limitations the lithography process play a main role. Physical limitations are the following: material properties, real values of refraction and absorption coefficients.

\section{Limits of Plane X-Ray Optics.}

Refractive index becomes more different from unit with increasing radiation wavelength. Greater thicknesses of the phase-shifting material are required for wave front phase modulation (Fig. 3), the phase difference of the waves diffracted on entrance and exit surfaces increasing. Under these conditions, the wave phase on the exit surface can no longer be regarded as known, and the plane optics approximation is not applicable. Besides, if the thickness of the optical elements is comparable with dimensions of the modulation zone, then re-reflected waves should be taken into consideration (see Fig. 3, dashed line). Thus, in optical elements fabrication the three-dimensional diffraction phenomena should be taken into account for the short wavelength radiation. The highest resolution attainable is restricted by the plane optics approximation.

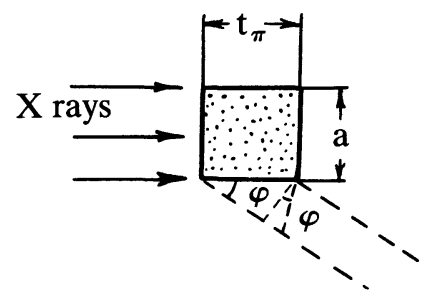

Fig. 3. - Additional phaseshift, due to the wave diffraction on exit and entrance surfaces of an optical element.

Using tabulated values of atomic scattering factors $f=f_{1}+i f_{2}$ [9], refractive index can be written as $1-\Delta n$, where

$$
\Delta n=\delta+i \beta=N_{\mathrm{a}} r_{\mathrm{e}} \lambda^{2} / 2 \pi\left(f_{1}+i f_{2}\right)
$$

here $N_{\mathrm{a}}$ is atom density, $r_{\mathrm{e}}$ is the electron radius, and $\lambda$ is wavelength. The thickness of the material $t \pi$ necessary for changing the transmitted radiation phase by $\pi$ equals $t \pi=\lambda / 2 \delta$. Let radiation scatter with an angle of $\varphi \operatorname{rad}(\varphi \ll 1)$, the phase difference of the waves scattered by entrance and exit surfaces of the element being equal to $\frac{\pi}{\lambda} t \varphi^{2}$ (see Fig. 3). The element can be considered flat as long as the phase difference does not exceed $\pi / 3$. Thus, the spatial frequency spectrum in plane X-ray optics is bounded by the value of $\varphi_{\min }=\frac{1}{2}\left(\frac{\lambda}{t \pi}\right)^{1 / 2}$, and the ultimate resolution is

$$
a_{\min }=2(\lambda \cdot t \pi)^{1 / 2}=\lambda /(2 \delta)^{1 / 2} .
$$

Hence, for wavelengths with $\frac{t \pi}{\lambda} \gg 1, a_{\min }$ decreases proportionally with decreasing $\lambda, a_{\min }=\varphi / \lambda$ remaining constant. This suggests that for these wavelengths the effects of three-dimensional diffraction are a factor that should be taken into account when producing optical elements in this wavelength range. For opaque modulation zones $t \pi=t^{*}$ is determined by the maximum thickness of the layer with a contrast that is sufficient to reach a reasonable diffraction efficiency $t^{*} \beta / \lambda \simeq 1$. Figure 4 shows a typical dependence $a_{\min }(\lambda)$. The scope of application

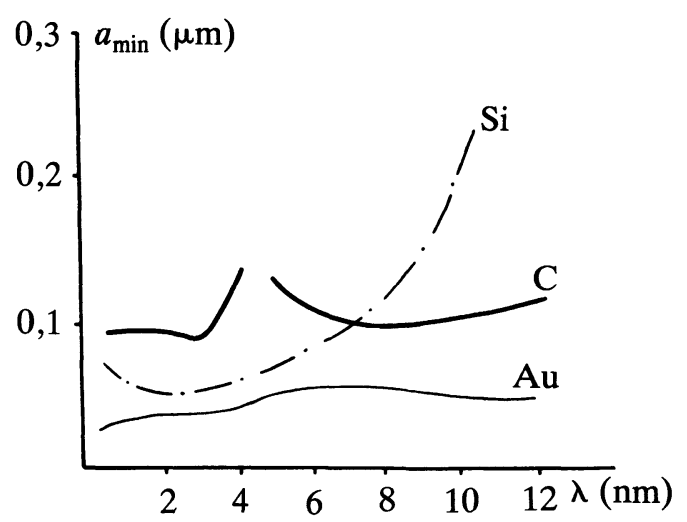

Fig. 4. - Maximum resolution $\left(a_{\min }\right)$ as a function of a wavelength for different elements.

of plane optics approximation is bounded by the highest resolution $a_{0} 30-50 \mathrm{~nm}$ attainable for various elements. To achieve a high resolution, it is necessary to change over from plane to three-dimensional Fresnel zones [10]. In this case, resolution is bounded by the technological limit of the fabrication of the Fresnel structure final zone, $\sim 15-30 \mathrm{~nm}$. Using curved (for instance, spherical) substrates or small angles of incidence on flat substrates, the resolution of X-ray optics elements can be increased by projecting the focal spot on the optical axis of the system. 
Three-Dimensional Optics with Multilayer X-Ray Mirrors.

Multilayer interference X-ray mirrors offer possibilities for fabrication of three-dimensional high resolution optical elements in the nanometric wavelength band (0.5-10 nm). Thus, Barbee and Underwood [11] have designed an X-ray microscope in accordance with the Kirkpatrick-Baez scheme, using multilayer coating to increase the reflection efficiency and radiation incidence angles. Though, when approaching normal incidence aberrations decrease, the need for a precise aligment of two spherical (cylinder) mirror does not enable resolution to be achieved.

The single-mirror scheme has also been studied by Barbee and Underwood [12]. Sharp images were obtained at the wavelength of $4.5 \mathrm{~nm}$ with the help of a spherical concave mirror made of a curved silicon plate with multilayer coating. However, the technique of spherical mirror fabrication by mechanical deformation of the plane silicon plate cannot exhibit high quality. Gaponov et al. [13] have taken a different line. They applied multilayer coating to a specially prepared, well polished, glass spherical substrate with a $500 \mathrm{~mm}$ radius. The experiment was carried out without radiation collimation, and the focal spot of 0.9-0.6 mm was completely determined by the synchrotron radiation beam divergence of $(1-2) \cdot 10^{-3} \mathrm{rad}$. Spiller et al. of IBM have succeeded in designing an X-ray scanning microscope with the wavelength of $68 \AA$ employing multilayer optics $[14,15]$. A similar instrument for wavelengths of about $20 \mathrm{~nm}$ has been developed by Haelbich of the Hamburg University [16].

The scheme at normal incidence can develop a high resolution, but a certain deviation from the normal incidence angle is required for separating the incident and the reflected beams. For a spherical substrate, it causes aberrations which do not permit bringing resolution to the diffraction limit. Geometrical aberrations can be taken into account and eliminated by forming a structure with a prearranged shape along multilayer coating surface. Using Bragg reflection from a multilayer mirror, one can modulate the exit wave in order to achieve radiation focusing diffraction correction of aberrations.

\section{Principles of Three-Dimensional Bragg-Fresnel Op-} tics.

A perfect Brag-Fresnel lens is a three-dimensional system of isophase surfaces rotational ellipsoides reflecting a spherical wave from point $A_{1}$ to point $A_{2}$ (Fig. 5). The distance between the surfaces is determined such that a path difference for radiation of a wavelength $\lambda$ reflected to point $A_{2}$ is multiple of $\lambda$. This perfect lens has a structure similar to a threedimensional hologram the properties of which are

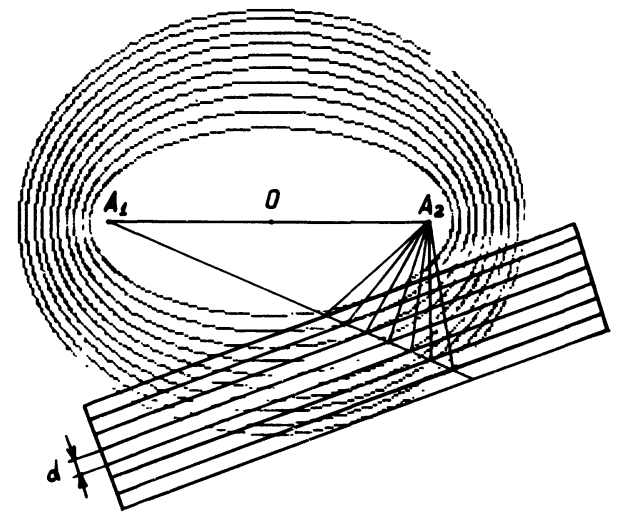

Fig. 5. - Scheme of volume Fresnel zone plate formation (when cutting surfaces of an equal phase by a multilayer structure with the $d$ period).

dictated by the behaviour of three-dimensional diffraction [17] :

- the resolution and shape of a diffraction maximum are determined by Laue three-dimensional function :

- each surface reflects an incident wave to the focal point $\mathrm{A}_{2}$ independently of radiation wavelength $\lambda$. Hence, chromatic aberrations in a perfect Bragg-Fresnel lens are absent ;

- the vision field of a lens is small and determined by its aperture. The distance between the variation source and the lens along the axis $\mathrm{OA}_{1}$ can vary within fairly wide limits. Therewith, the distance $\mathrm{OA}_{2}$ changes as in conventional zone plate optics ;

- the displacement of reflecting plates towards each other by the value $\Delta d$ in the lens adjacent regions leads to phase changes of waves scattered by these regions by a value proportional to $\frac{\Delta d}{d} 2 \pi$, where $d$ is the distance between surfaces. This allows for controlling reflected beams and for their immediate switching.

Any real X-ray optical element is more or less a good approximation of such perfect structure. Below we consider the approximation of a shaped multilayer structure deposited on a plane, parabolic or spherical substrates.

The significant distinction of Bragg-Fresnel optics lies in the fact that an image is formed in the Fresnel diffraction region, i.e. diffraction is described with consideration for quadratic members in low-angle approximation of the Fresnel-Kirchhoff integral [18]. In contrast, the conventional description of diffraction on multilayer structures takes into account only the Bragg condition in a plane-wave approximation. In fact, this description considers the model of an infinite one-dimensional diffraction lattice regarding the effects of dynamic diffraction in absorption conditions and the imperfection of an interface surface shape. 
The description of X-ray spherical wave diffraction with consideration for the members of the second order in disintegration for periodic lattices in crystals was made in [19].

For a spherical wave source situated at an ultimate distance $R_{1}$ from a multilayer structure, cophased scatterers are located in corresponding volume Fresnel zones. Figure 6 shows the interaction of Fresnel volume zones, a point source and its image with a perfect Bragg lattice for a symmetrical case of distance equality up to the source and its image $\left(R_{1}=R_{2}\right)$. The angle $\theta$ corresponds to the Bragg reflection angle from multilayer structure with a period $d$. Actually, the image in figure 6 is that of a Fresnel volume zone plate with a clearly defined change in a zone period in the depth of a multilayer structure. In fact, a complete three-dimensional structure of Fresnel zones must be taken into account when the radiation extinction depth exceeds the size of the first zone. It is typical of certain subnanometer radiation reflection in crystals as well as of neutron diffraction. For multilayer structures in the nanometer wavelength range the number of layers $N$ does not exceed a few tens and is much smaller than their number in the first Fresnel zone. The inclination of zone sections is negligibly small within the rocking curve. Such optical element with direct zones in the aperture of the angles of the rocking curve is similar to a standard plane diffraction optical element. The image is formed in a few orders of diffraction (at different focal distances). But three-dimensional zone plates with bending zones display all the properties of thick holograms

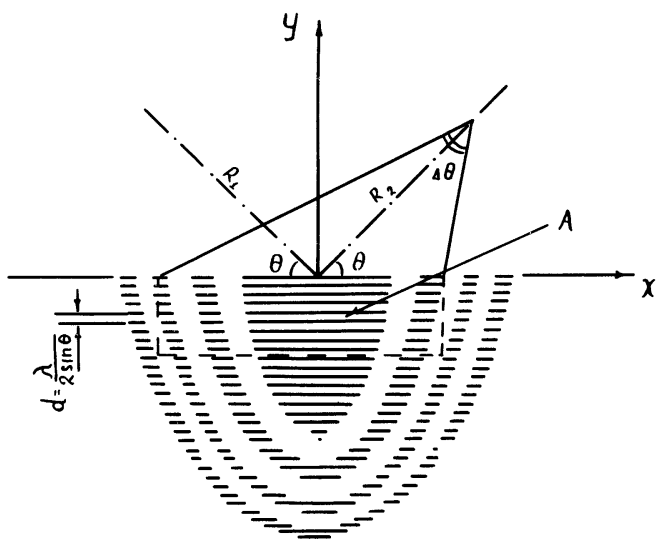

Fig. 6. - View of Fresnel zones. $\Delta \theta$ is the width of a rocking curve of Bragg diffraction. $A$ is the region of Bragg diffraction. and, thus, form nonaberration monochromatic images. It is obvious that the angular aperture of an optical element in this case is much greater than the width of the rocking curve $\Delta \theta$. Reflection for such a structure exists at any angle, any large width of the rocking curve and the concept of the angular rocking curve itself, introduced for plane waves, has no sense. Two possibilities for fabricating thick optical elements follow from the structure of Fresnel volume zones (Fig. 6). The first is hard X-ray diffraction $(<0.5 \mathrm{~nm})$ on crystals. In this case the extinction depth is usually much greater than the first Fresnel zone and permits fabricating a volume Fresnel structure. The second is soft $\mathrm{X}$-ray diffraction $(0.5 \mathrm{~nm}<\lambda<10 \mathrm{~nm})$ on multilayer interference mirrors [20]. As a result of high absorption and a great reflection coefficient from each layer, the number of reflecting plates is small $(N \sim 100 \ll$ the number of plates in the first Fresnel zone). Here, modulation along the surface is required. These optical elements are very similar to thick holograms of the visible wavelength range where the number of reflecting plates is also small.

It is of interest that Fresnel zones can be straightened out within a wide aperture at the cost of fabrication of multilayer mirror nonperiodic layers. This phenomenon significantly simplifies the fabrication of an optical element since there is no need to fabricate a complex bending zone. Figure 6 shows a Fresnel zone structure for a symmetrical case $R_{1}=R_{2}$ on periodic layer and the shape of the same structure on nonperiodic layers (Fig. 7). This effect was first described by Shulakov and Aristov [21].

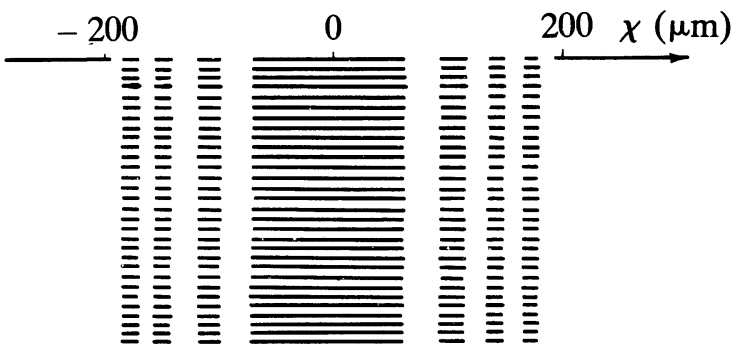

Fig. 7. - Fresnel zones straightened out at the cost of nonperiodic alternation of reflecting layers.

Let us analyse the shape of the zone structure for section in the plate $z=0$ (Fig. 6). The path difference between the point at the origin of coordinates and an arbitrary point $(x, y)$ which appears in Fresnel integral equals

$$
\Delta l(x, y)=\left(\left(x-x_{1}\right)^{2}+\left(y-y_{1}\right)^{2}\right)^{1 / 2}-\left(x_{1}^{2}+y_{1}^{2}\right)^{1 / 2}+\left(\left(x_{1}+x_{2}\right)^{2}+\left(y_{1}-y_{2}\right)^{2}\right)^{1 / 2}-\left(x_{2}^{2}+y_{2}^{2}\right)^{1 / 2}
$$

where $x_{1} y_{1}$ are the coordinates of the source point, $x_{2} y_{2}$ are the coordinates of the image point

$$
\begin{aligned}
& R_{1}=\left(x_{1}^{2}+y_{1}^{2}\right)^{1 / 2} \\
& R_{2}=\left(x_{2}^{2}+y_{2}^{2}\right)^{1 / 2} .
\end{aligned}
$$


By expanding the expression under the integral sign of Fresnel integral into a series $\left(x, y \ll R_{1}\right.$ and $R_{2}$ ) and performing appropriate transformation we obtain

$$
\begin{aligned}
& \Delta l(x, y)=\frac{1}{2 R_{1}}\left\{x^{2}+y^{2}-2 x x_{1}-2 y y_{1}\right\}+\frac{1}{2 R_{2}}\left\{x^{2}+y^{2}-2 x x_{2}-2 y y_{2}\right\} \\
& -\left[\frac{1}{8 R_{1}^{3}}\left(4 x^{2} x_{1}^{2}+4 y^{2} y_{1}^{2}+8 x y x_{1} y_{1}\right)+\frac{1}{8 R_{2}^{3}}\left(4 x^{2} x_{2}^{2}+4 y^{2} y_{2}^{2}\right.\right. \\
& \left.\left.+8 x y x_{2} y_{2}\right)\right]+\left[\frac{1}{8 R_{1}^{3}}\left(4 x^{3} x_{1}+4 y^{3} y_{1}+4 x^{3} y y_{1}+4 y^{2} x x_{1}\right)\right. \\
& \left.+\frac{1}{8 R_{2}^{3}}\left(4 x^{3} x_{2}+4 y^{3} y_{2}+4 x^{2} y y_{2}+4 y^{2} x x_{2}\right)\right] \\
& -\left[\frac{1}{8 R_{1}^{3}}\left(x^{4}+y^{4}+2 x^{2} y^{2}\right)+\frac{1}{8 R_{2}^{3}}\left(x^{4}+y^{4}+2 x^{2} y^{2}\right)\right] .
\end{aligned}
$$

According to Meier [22] the first term in the square brackets corresponds to astigmatism, the second to koma and the third to spherical aberration. The curvatures of the field and distortion are taken into account in the first two terms in the brace brackets, since the expansion into a series was performed in terms of $R_{1}$ and $R_{2}$ rather than $R_{1}^{\prime}=R_{1} \sin \theta$ and $R_{2}^{\prime}=R_{2} \sin \theta$, where $\theta$ is the angle of incidence and reflection (the Bragg angle). The term corresponding to spherical aberration can be neglected. Since for mirrors $y \ll x(y<1 \mu \mathrm{m})$, from all the terms corresponding to koma only the terms at $x^{3}$ have a value. Moreover, as $-\frac{x_{1}}{R_{1}}=\frac{x_{2}}{R_{2}}$, the Bragg term $-x\left(\frac{x_{1}}{R_{1}}+\frac{x_{2}}{R_{2}}\right)=0$, i.e. there is Fresnel diffraction along the $x$-axis and Bragg diffraction along the $y$-axis. As a whole diffraction on such a structure can be considered as Bragg-Fresnel diffraction. With allowance made for the foregoing we have

$$
\begin{aligned}
\Delta l(x, y)= & -y\left(\frac{y_{1}}{R_{1}}+\frac{y_{2}}{R_{2}}\right)+\left(x^{2}+y^{2}\right)\left(\frac{1}{2 R_{1}}+\frac{1}{2 R_{2}}\right) \\
& -y^{2}\left(\frac{y_{1}^{2}}{2 R_{1}^{3}}+\frac{y_{2}^{2}}{2 R_{2}^{3}}\right)-x^{2}\left(\frac{x_{1}^{2}}{2 R_{1}^{3}}+\frac{x_{2}^{2}}{2 R_{2}^{3}}\right) \\
& -x y\left(\frac{x_{1} y_{1}}{R_{1}^{3}}+\frac{x_{2} y_{2}}{R_{2}^{3}}\right)+x^{3}\left(\frac{x_{1}}{2 R_{1}^{3}}+\frac{x_{3}}{2 R_{2}^{3}}\right)
\end{aligned}
$$

According to the formula of the lens $\frac{1}{R_{1}}+\frac{1}{R_{2}}=\frac{1}{F}$, where $F$ is a focal distance. Since $-x_{1} / R_{1}=$ $x_{2} / R_{2}=\cos \theta, y_{1} / R_{1}=y_{2} / R_{2}=\sin \theta$ we obtain

$$
\begin{aligned}
\Delta l(x, y)= & -y \sin \theta+y^{2} \frac{1}{2 F} \cos ^{2} \theta+x^{2} \frac{1}{2 F} \sin ^{2} \theta \\
& +x y \cos \theta \sin \theta\left(\frac{1}{R_{2}}-\frac{1}{R_{1}}\right)+x^{3} \cos \theta\left(\frac{1}{R_{2}^{2}}-\frac{1}{R_{1}^{2}}\right) .
\end{aligned}
$$

The first term in (8) describes Bragg diffraction $2 y \sin \theta=\lambda$. The second term shows the value of the first Fresnel zone at $x=0: y_{1}=(\lambda F)^{1 / 2} / \cos \theta$. By dividing $y_{1}$ into an interplanar distance $y=\frac{\lambda}{2} \sin \theta$ for the number of Bragg planes in the first Fresnel zone we obtain

$$
N_{1}=2(F / \lambda)^{1 / 2} \operatorname{tg} \theta .
$$

For $\lambda=5 \mathrm{~nm}$ and $F=5 \mathrm{~cm}, N_{1}=10^{4} \operatorname{tg} \theta$, and for a real structure $(N \sim 100)$ volume Fresnel diffraction manifests itself only at $\theta<1^{\circ}$. At incidence angles of radiation $\theta>10^{\circ}, N_{1} \sim 10^{3}-10^{4}$, i.e. the zones in the central part at the depth $N=100$ are straight. They start bending only when they are located far from the centre along the surface. The third term in expression (8) describes Fresnel zones for $y=0$. These zones are similar to a plane zone plate at a normal incidence angle $x_{N}=(N F \lambda)^{1 / 2}$, but the focal distance for the same zone sizes decreases as $F=F_{0} \sin \theta$. If the angle of incidence $\theta<10^{\circ}$, the last term in expression (8) exceeds the third one and the zone shape along the surface is complex.

The first experiments on X-ray radiation focusing on a Bragg-Fresnel optical element were performed on a multilayer interference structure Ni-C [23]. A 
profile for amplitude of the X-ray wave front was fabricated in a multilayer interference mirror. For phase modulation a gold layer of $\sim 300 \AA$ thick was deposited on the multilayer mirror surface along even Fresnel zones. An X-ray tube with a linear focus and $\mathrm{Fe}$ anode was used as a source of X-ray radiation. A slit of $\sim 50 \mu \mathrm{m}$ is placed behind the tube. An optical element is fixed in a goniometric head at a distance of $18 \mathrm{~cm}$ from the slit. When the central zone is $180 \mu \mathrm{m}$ and the angle of incident on the mirror surface is $1.3^{\circ}$, the demagnified image of the slit was observed at a distance of $1.7 \mathrm{~cm}$ from a focusing element. Holographic plates (Mikrat) with a self resolution of $\sim 0.2 \mu \mathrm{m}$ were used as a recorder. Figure 8 gives a densitogram of the slit image in the region of the spectrum typical line $\mathrm{FeK} \alpha$. The size of the image slit $\sim 50 \mu \mathrm{m}$ corresponds to a demagnification in the optical experimental scheme. For radiation with a wavelength of $0.154 \mathrm{~nm}$ focusing was obtained on a $\mathrm{Si}$ profiled crystal in the same experimental arrangement [24, 25]. Figure 9 shows the ellipsoidal zone plate made from multilayer mirror by electron-beam lithography and ion-beam etching.

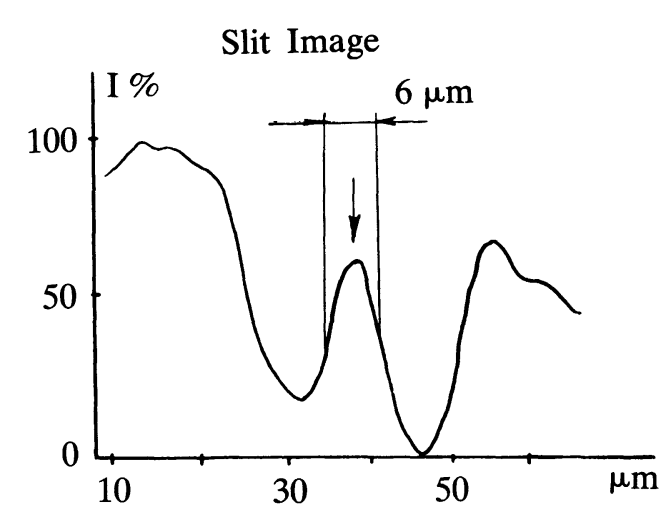

Fig. 8. - Densitigram of the slit image (pointed by an arrowed line) in focusing on a profiles multilayer mirror $(\lambda=1.96 \AA)$.

Volume diffraction of $\mathrm{X}$-ray radiation gives a possibility not only to perform beam focusing but also to modulate it with space and time. For example, interaction between an X-ray wave and a surface - acoustic wave propagating along a multilayer structure permits obtaining rather strong diffraction reflex. Spatial position of this reflex depends on the frequency of an acoustic wave. Figure 10 shows intensity distribution in the far field of diffraction during reflection from a multilayer mirror modulated by the surface acoustic wave [26]. At the amplitude of a surface deformation of $0.4 \mathrm{~nm}$ the efficiency of a diffraction order on the wavelength $0.196 \mathrm{~nm}$ was $\sim 10 \%$.

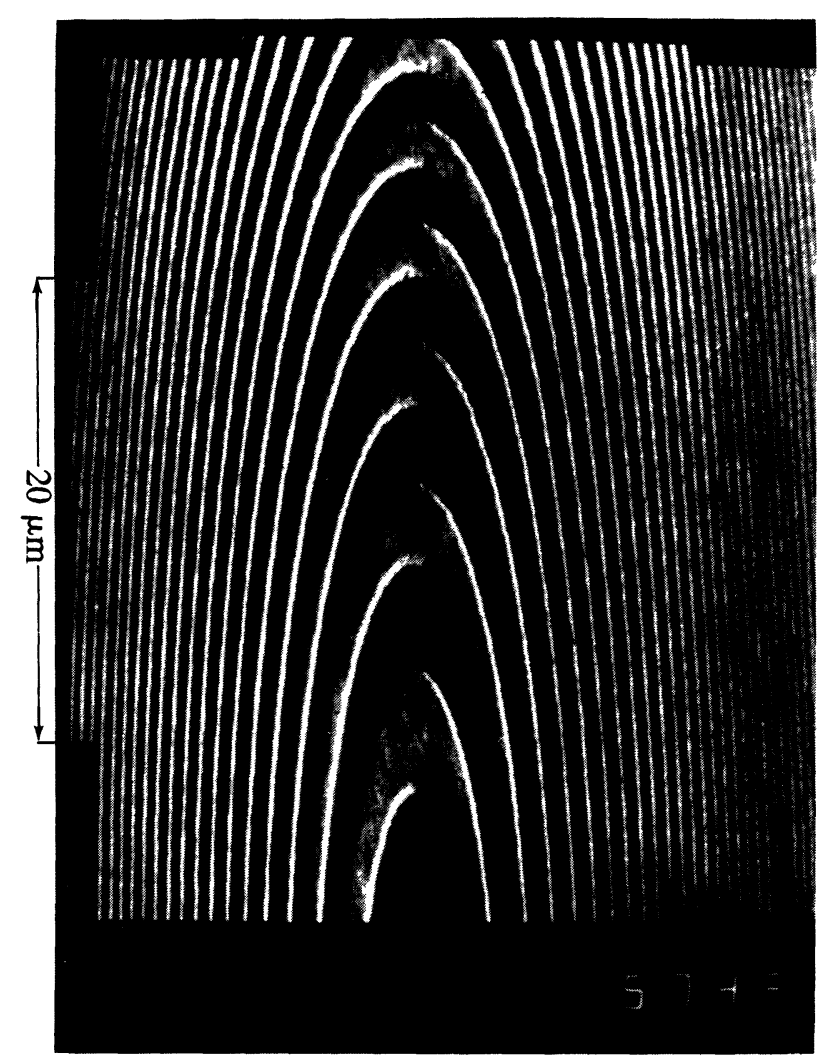

Fig. 9. - Bragg-Fresnel optical element made from a multilayer mirror by electron-beam lithography, and ionbeam etching.

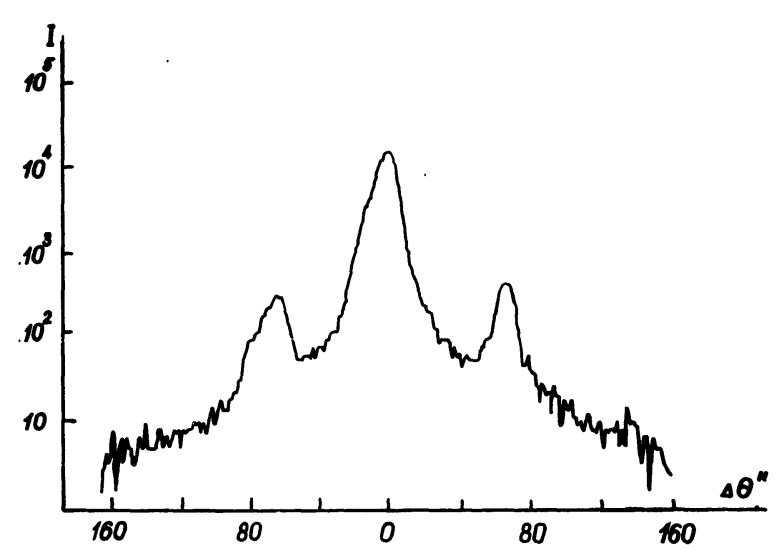

Fig. 10. - Radiation intensity distribution in the far field in diffraction on a multilayer structure modulated by an acoustic wave.

\section{Conclusion.}

The achievements in microstructure fabrication technology permit the resolution of plane optical elements based on Fresnel zone plates to approach a theoretical resolution limit caused by the effects of volume diffraction. The latest advances in multilayer interference structure fabrication, microstructuring technology and in fabrication of smooth surfaces of a given form enable us to reach a wavelength up to 
$\lambda \sim 0.5 \mathrm{~nm}$ and to make the theoretical resolution limit closer to the value of a wavelength through designing volume optical elements with allowance made for the effects of three-dimensional diffraction. The results obtained for $\mathrm{X}$-ray radiation focusing $(\lambda \sim 0.2 \mathrm{~nm})$ on a profiled multilayer mirror are expected to ensure progress in the development of $\mathrm{X}$-ray optics. X-Ray optical elements on profiled multilayer mirrors are likely to find increasing application in high resolution image transmission in scanning microscopy and photoelectron spectroscopy. They can also be used as highly efficient focusing monochromators of an X-ray wavelength range.

Small angles of incidence on plane profiled mirrors are most effective for the range of $\mathrm{X}$-ray radiation with a short wavelength $(\sim 0.5 \mathrm{~nm})$. Since the angles are very small, the effect of aberrations is very profound and can be eliminated for one point by calculating a profile in a multilayer coating with regard to aberration. However, the visual field is very small under these conditions. Hence, such optical elements can be used to advantage when only radiation focusing is required, for instance, for scanning microscopy or for scanning photoelectron spectroscopy. The application of profiled mirrors on spherical substrates at incidence angles close to normal $\left(\theta \sim 80^{\circ}\right)$ is promising for the soft wavelength range $\lambda \sim 5 \mathrm{~nm}$. Aberrations at such angles are small and can be almost completely eliminated by means of submicron technology for a greater visual field. Optical elements for nearly normal incidence show promise when used for image transmission.

\section{Acknowledgments.}

We thank S. V. Gaponov, N. N. Salashchenko for useful discussion of this work, as well as $\mathrm{N}$. V. Gornakova for microlithography process.

\section{References}

[1] Kirkpatrick, P., BAEZ, A. V. 38 (1948) 766.

[2] Jentzsch, F., Phys. Z. 30 (1929) 268.

[3] Wolter, H., Ann. Phys. 10 (1952) 94, 286.

[4] BAEZ, A. V., JOSA 51 (1961) 405 ; 42 (1952) 756.

[5] Ceglio, N. M., AIP Conf. Proc. N 75 (1981) 210.

[6] Kirz, J., Rarback, H., Rev. Sci. Instrum. 56 (1) (1985) 1

[7] Rudolph, D., Schmahl, G., Ann. N.Y. Acad. Sci. 342 (1980) 94.

[8] Underwood, J. H., Atwood, D. T., Phys. Today (April 1984) 44.

[9] Henke, B. L. et al., AIP Conf. Proc. 75 (1981) 340.

[10] ARISTov, V. V., Shulakov, E. V., Kristallografiya (1987) in press.

[11] Underwood, J. H., Barbee, T. W., Appl. Opt. 25 (1986) $11,1730$.

[12] Underwood, J. H., BarbeE, T. W., Nature 294 (1981) 429.

[13] Gaponov, S. V., Gusev, S. A., Platonov, Yu. Ya., Salashchenko, N. N., Gluskin, E. S., Pisma Zh. Eksp. Teor. Fiz. 9 (1983) 4, 208.

[14] Lovas, I., et al., Proc. SPIE 316 (1980) 90.

[15] SPILlER, E., Scanned Image Microscopy, Ed. E. Ash (Academic, New York) 1980, p. 369.

[16] HaElbich, R., Scanned Image Microscopy, Ed. E. Ash (Academic, New York) 1980, p. 413.

[17] Aristov, V. V., SheKhtman, V. Sh., Usp Fiz. Nauk 104 (1971) 1, 51 (in Russian).
[18] Cowly, J. M., Diffraction Physics (North-Holland, Amsterdam) 1975.

[19] Shulakov, E. V., Aristov, V. V., Acta Crystallogr. A 38 (1982) 454.

[20] Ceglio, N. M., Stearins, D. G., Hawryluk, A. M., Applications of Thin-Film Multilayered Structures to Figured X-Ray Optics, SPIE 563 (1985) 360-366.

[21] Aristov, V. V., Shulakov, E. V., Preprint, Chernogolovka (1987) ; Opt. Commun. (to be published).

[22] MeIER, R. W. 55 (1965) 987.

[23] Aristov, V. V., Gaponov, S. V., Genkin, V. M., Gorbatov, Yu. A., Erko, A. I., Martynov, V. V., Matveeva, L. A., SalashchenKo, N. N., Fraerman, A. A., JETP Lett. 44 (1986) 4, 207.

[24] Aristov, V. V., Basov, Yu. A., Snigirev, A. A., Pisma Zh. Eksp. Teor. Fiz. 13 (1987) 2, 144 (in Russian).

[25] Aristov, V. V., Snigirev, A. A., Basov, Yu. A., Nikulin, A. Yu., AIP Conf. Proc. 147 (1986) 253.

[26] Aristov, V. V., Vereschagin, G. V., Erko, A. I., Matveeva, L. A., Roschupkin, D. V., Pisma Zh. Eksp. Teor. Fiz. 13, 21 (1987) 1288 (in Russian). 